\section{The use of a visual mask may seriously confound your experiment}

\author{
CHARLES W. ERIKSEN \\ University of Illinois at Urbana-Champaign \\ Urbana, Illinois 61801
}

A wide range of visual information processing experiments have in common the procedure of brief stimulus exposure durations. When brief exposure durations are used, it has become almost standard operating procedure to use a visual noise mask. In fact, not only is the stimulus followed by a mask (backward masking), but it is also frequently preceded by a mask as well (forward masking). Seldom, however, does the experimenter provide a rationale for the use of these masking procedures or an explicit account of what he thinks the masks have accomplished in terms of changing the nature of the visual input. Along with this absence of rationale, there is a neglect of control procedures to demonstrate that the mask was effective (although, in the absence of rationale, it is difficult to answer this latter question). Thus, we are left with the uncertainty as to whether the mask had any effect upon the visual input, whether it affected all stimuli equally, and whether the results of the experiment would have been any different if masking procedures had not been employed.

One ostensible reason why an experimenter might employ a masking procedure is to degrade the stimulus so as to control the level of the subject's performance to a range somewhere between chance levels and $100 \%$. But there are better methods available to achieve these goals, methods that are more direct and with less uncertainty as to their effects. Exposure duration can be manipulated, which directly affects the stimulus energy, or, alternatively, the energy can be manipulated by varying the luminance. Should these methods be inappropriate to the general experimental situation, the target forms can be blurred or the figure-ground contrast varied. As contrasted with masking, these procedures have the desirable characteristic of allowing a clear specification of just what the stimulus was that was available to the visual system.

While some of the experimenters who employ masking techniques do so for no more articulated reason than that others have done so, I suspect that most of the investigators use masks to overcome the

This research was supported by U.S. Public Health Service Research Career Program Award K6-MH-22014 and by Public Health Service Research Grant MH-01206. problem of stimulus persistence, or the icon. This iconic persistence is inconvenient if one wishes to specify precisely just how long a visual stimulus was available for input processing. It precludes the simplifying assumption that the stimulus was available only for the time that it was physically presented. Many experimenters who employ the masking procedure apparently assume that the occurrence of the mask effectively terminates input processing of the stimulus. According to this assumption, if the stimulus was presented for $30 \mathrm{msec}$, followed immediately by a noise mask, then whatever information the subject derived from the stimulus must have occurred during those $30 \mathrm{msec}$ of input. But, how secure is this assumption?

A primary requirement of sound experimental technique is that the experimental manipulations be clearly understood, unambiguous, and noncontroversial as to their effects. Otherwise, the results of the experiment will be confounded in interpretation by the varying assumptions as to the action of the manipulation. Visual masking does not meet these criteria. The mechanisms underlying masking phenomena are still under investigation, and several different theories have been advanced. Two general, but quite different, theoretical explanations have been advanced for visual masking (Felsten \& Wasserman, in press): interruption masking and integration masking. Only the former is reasonably compatible with the assumption that a mask, following stimulation, effectively limits the time available for stimulus processing. Interruption theories, of which there are several versions (Spencer \& Shuntich, 1970; Turvey, 1973), have in common the assumption that a second arriving stimulus takes priority in processing and thus diverts the processing mechanisms from the first stimulus to the mask.

Integration theories take as their point of departure the well-documented lack of fine temporal resolution of the visual system (Eriksen \& Schultz, 1978; Felsten \& Wasserman, in press; Schultz \& Eriksen, 1977). When two successive visual stimuli are separated by only a short temporal interval, they are summed together into a composite, resulting in a montage-like effect. Recognition performance is impaired due to this degrading of the stimulus by the superimposition of the mask. The temporal interval over which this summation or integration occurs depends upon several variables, such as luminance levels of the stimuli and the mask and the state of adaptation of the eye, but measurable effects can be obtained over stimulation durations in excess of $150 \mathrm{msec}$.

The evidence for integration theories is impressive 
and substantial (Eriksen \& Schultz, 1978; Felsten \& Wasserman, in press), so much so that recent advocates of interruption theories have incorporated integration into their accounts of masking (Helliger, Walsh, Lawrence, \& Prassey, 1979; Spencer \& Shuntich, 1970; Turvey, 1973). In these accounts, integration masking is recognized as occurring at shorter stimulus onset asynchronies (as long as $150 \mathrm{msec}$ ) and interruption masking as occurring at longer asynchronies.

The implications of integration masking for visual information processing experiments are quite different from those of interruption masking. In experiments that employ brief exposure durations for the stimulus, followed immediately by the mask, the stimulus onset asynchrony is well within the interval that even interruption theorists recognized as resulting in integration masking. Thus, the assumption that the input processing time is limited to the duration of the stimulus is highly questionable. It may well be that the image of the mask superimposed upon the stimulus trace of the preceding stimulus degrades that trace to a point at which no further information can be extracted from it. But, if so, it is contingent upon the experimenter to show by control procedures that this is indeed the case.

One control would be to simultaneously present the target and the mask and to show that target identification accuracy is at chance levels. This is only the bare minimum control. It does not ensure that when the mask is delayed by $30 \mathrm{msec}$ or more, the mask occurrence prevents further information extraction from the target stimulus. Sufficient information could have been extracted about the target during the $30 \mathrm{msec}$ of unimpaired target duration to serve as cues for further information extraction after the mask is superimposed upon the target. The simultaneous occurrence of target and mask may render the target undecipherable, but, given a few clues as to its nature-cues that become available during the first few milliseconds of unimpaired exposure-the otherwise unintelligible montage could yield further information concerning the target's identity.

If integration of the target and the mask only made uncertain the amount of time the target was available for extracting information, the use of masking procedures in visual information processing experiments might be only an unnecessary procedural complication. But, the use of masks creates all sorts of mischief. Those who have experimented with different types of noise masks have found that the masks differ in their effectiveness. What is not so widely considered, however, is the fact that the same mask will differ in its effectiveness for different form stimuli. The same mask that impairs recognition of one target can actually enhance recognition of a different target (Schultz \& Eriksen, 1977). When the possibility of integration of the target and the mask is considered, it is readily apparent that the degree to which target recognition is impaired depends upon which details of the mask are superimposed upon which details of the target. When a tachistoscope is used, as opposed to a CRT display, there is the additional consideration that the contrast of some of the target's details are enhanced when parts of the mask coincide with their outlines. Some experimenters take the laudable precaution of selecting sets of target letters based upon data from confusion matrices (Gilmore, Hersh, Caramazza, \& Griffin, 1979; Townsend, 1971), but then vitiate these efforts by neglecting to determine whether these letters remain equally recognizable and confusable when followed by the chosen mask. ${ }^{1}$

A given mask is apt to vary in its effectiveness for different target forms, and when the experimental apparatus is a T-scope, further noise variance may be introduced. This is a result of the slop that is nearly always present in the stimulus card or slide holders for the T-scope. One can readily verify this by taking a typical noise mask constructed from bits and pieces of letters (or other favorite masks such as a dollar or number sign), placing it in one field of the T-scope, and placing a target letter in the other field. Now, activate both fields simultaneously for a severalsecond duration. As the two cards or slides are manipulated to move around in the play provided by the respective holders, the identifiability of the target letter generally will be found to vary appreciably. During the course of running an experiment, this type of misalignment occurs randomly from trial to trial and adds an unnecessary amount of error variance to the data.

The lack of precision and alignment of target detail relative to mask detail not only increases error variance, but also makes it exceedingly difficult to closely replicate the conditions of a reported experiment. This difficulty is compounded when a noise mask consists of bits and fragments of letters or other forms randomly distributed. The random arrangement of this "noise" makes it virtually impossible to determine which fragments of the mask superimpose upon which details of the target form.

Irrespective of theoretical interpretation, it is patently clear that visual masking is not all-or-none phenomenon. By this, I mean it is possible to vary the target-mask energy ratios and also the character of the mask so that the mask's effect on the target at a given SOA varies from no observed effect at all (target identification $100 \%$ ) to reducing target identification to the chance level. It might seem that this observation would embarrass interruption theorists unless they wish to consider the possibility of "partial interruption." But, there is a more direct way out of this embarrassment. The relative strength of the mask can be considered to reflect the proportion of trials in 
which it will interrupt processing. Thus, a mask that was relatively weak in relation to the target would interrupt processing on only a small proportion of the trials, but, when the interruption occurred, it would be complete.

Either interpretation, however, poses problems for the use of masking to control input processing time. If partial interruption is invoked, then the mask has not stopped input processing of the target, but only impaired it. If the probability interpretation is preferred, then the mask limited processing only on some undetermined proportion of the trials. When the experimenter does not provide data as to the effectiveness of his masking manipulation, we can only guess as to how partial it was or, alternatively, on what proportion of the trials it interrupted processing.

For many, if not most, visual information processing studies, the need to control input processing time is not clear. There is no rationale provided to leave one to expect different results if the desired level of accuracy for the experiments was obtained by shortening the exposure duration, lowering the luminance, or reducing the figure-ground contrast instead of backward masking. What difference does it make if the performance level is partly due to processing from the stimulus trace or decaying icon, as opposed to the assumption that all the information that the subject receives is obtained during the $\mathrm{n}$ msec when the physical stimulus was available?

To clarify this point, consider an example. Assume a small set of target letters that are individually exposed in a tachistoscope. Assume further that identification accuracy is $75 \%$ with a 10 -msec exposure duration. Now we decide that we want to backward mask these stimuli with a given random noise mask that onsets with target termination. Other things being equal, we would find that the exposure duration has to be increased appreciably in order to maintain $75 \%$ accuracy. Let us say that the new duration with mask is $30 \mathrm{msec}$. In terms of conventional thinking, in the nonmasked situation, the visual system and brain extract information during the $10 \mathrm{msec}$ when the stimulus is actually present and for an unmeasured $\mathrm{n}$ msec from a decaying trace or icon. In the masking paradigm, assuming interruption, the same level of information is extracted during the $30 \mathrm{msec}$ when the stimulus is present, yet here the visual system receives three times as much energy. But, do these two situations differ as far as subsequent processing stages or mechanisms are concerned? Since the amount of information extraction is the same ( $75 \%$ accuracy) with either the masking or the nonmasking procedure, does it make any theoretical difference which procedure is used?

It may, if we are attempting to solve the knotty problem of perceptual rate, but for most information processing research, it probably doesn't. Few, if any, models are so explicitly detailed as to distinguish between masked and nonmasked inputs, and the formulations they pose for experimental tests would be the same with either.

Illustrative of this latter point is recent research on the interactive channels model as applied to visual search (Estes, 1974). These experiments have been concerned with testing whether the recognition accuracy for target letters in a display is affected by the degree of feature similarity or featural overlap the target shares with noise letters in the display. In several of these experiments (Bjork \& Murray, 1977; Santee \& Egeth, 1980), the visual display was presented for a brief duration such that target identification was above chance but well below $100 \%$. The display was followed immediately by a noise mask. The experimental manipulation consisted of varying the degree of featural overlap between the target letter and the noise in the display.

In these experiments, no justification was provided for the masking procedures. It is not evident that inhibition and interaction among input channels feeding to feature detectors would have been any less effectively tested if the overall performance level on targets in the experiments had been controlled by exposure duration alone, rather than by duration and a mask. In fact, the use of masks in these studies actually would seem to confound the interpretation of the effects of feature similarity, since the masking stimuli have an unspecified feature overlap with both the target and the noise letters. It is basically an empirical question as to whether similar results would be obtained if the mask were eliminated and the necessary impoverishment of the information in the displays were obtained by reducing the exposure duration, the luminance, or the figure-ground contrast. But, it is to be noted that the degree of explicitness of the theory under test does not yield predictions for differential effects from these different ways of impoverishing the information in the stimulus. Thus, why complicate the procedure?

There is probably a whole generation of cognitive psychologists who believe that stimuli cannot be presented in a tachistoscope unless the presentation is followed by a mask. Many will continue to use masking procedure when their experiments require brief exposure durations, but, if they do, there are certain obligations they should meet. The reasons for the masking procedure should be stated, and the experimenters should deal explicitly with how the mask affected their results and theoretical predictions as contrasted with other methods of impoverishing the stimulus inputs. Data should be provided to show not only that the mask was effective, but also how much it reduced performance over nonmasked presentation. 
And, most importantly, experimenters should demonstrate that the mask affected all their stimuli equally and uniformly.

\section{REFERENCES}

Bjork, E. L., \& Murray, J. T. On the nature of input channels in visual processing. Psychological Review, 1977, 84, 472-484.

Eriksen, C. W., \& Schultz, D. W. Temporal factors in visual information processing. In J. Requin (Ed.), Attention and performance VII. New York: Academic Press, 1978.

Estes, W. K. Redundancy of noise elements and signals in visual detection of letters. Perception \& Psychophysics, 1974, 16, 53-60.

Felsten, G., \& Wasserman, G. S. Visual masking: Mechanisms and theories. Psychological Bulletin, in press.

Gilmore, G. C., Hersh, H., Caramazza, A., \& Griffin, J. Multidimensional letter similarity derived from recognition errors. Perception \& Psychophysics, 1979, 25, 425-431.

Hellige, J. B., Walsh, D. A., Lawrence, V. W., \& Prassey, M. Figural relationship effects in mechanisms of visual masking. Journal of Experimental Psychology: Human Perception and Performance, $1979,5,88-100$.
Santee, J. L., \& Egeth, H. E. Interference in letter identification: A test of feature-specific inhibition. Perception \& Psychophysics, 1980, 27, 321-330.

Schultz, D. W., \& Eriksen, C. W. Do noise masks terminate target processing? Memory \& Cognition, 1977, 5, 90-96.

Spencer, T. J., \& Shuntich, R. Evidence for an interruption theory of backward masking. Journal of Experimental Psychology, 1970, 50, 198-203.

Townsend, J. T. Theoretical analysis of an aiphabetic confusion matrix. Perception \& Psychophysics, 1971, 2, 45-53.

Turvey, M. T. On peripheral and central processes in vision: Inferences from an information-processing analysis of masking with patterned stimuli. Psychological Review, 1973, 80, 1-52.

\section{NOTE}

1. It is to be noted that confusion data obtained when all 26 alphabet characters are alternatives do not necessarily generalize to a subset of the alphabet. It has been recognized for some time that the identifiability of a given form cannot be expressed independently of the possible alternatives.

\section{(Received for publication May 1, 1980;} accepted May 1, 1980.) 